\title{
PARAMETRIC HERITAGE. THE DIGITAL RECONSTRUCTION OF P.L. NERVI'S PALAZZO DEL LAVORO IN THE 3DMODELLING AGE
}

\author{
A. Tomalini ${ }^{1 *}$, M. Lo Turco ${ }^{2}$ \\ ${ }^{1}$ DAD, Department of Architecture and Design, Politecnico di Torino, Italy - andrea.tomalini@ studenti.polito.it \\ ${ }^{2}$ DAD, Department of Architecture and Design, Politecnico di Torino, massimiliano.loturco@polito.it
}

\author{
Commission II, WG II/8
}

KEY WORDS: HBIM, Algorithmic Design, Interoperable processes, P. L. Nervi

\begin{abstract}
:
One of the main themes of this work is to incorporate the geometric aspects that characterize a certain type of architecture within a broader rule system, which, in the whole, generate the forms. Therefore, the solution would be to draw the reconstruction of a parametric model, through the application to a selected case study: Palazzo del Lavoro of Pier Luigi Nervi. The identification of some weaknesses of the project is important in order to digitally intervene on them, to prefigure a new life of the artifact in a virtual environment. By doing so, it will be possible to have truthful feedback in the real environment. The survey phase did not intend to reason on the architecture in its complexity, but rather on the observation of the matrices that regulate the similar geometries between them. The translation of these matrices into mathematical models, following the logic of HBIM and Algorithmic Design (AD), has allowed the creation of two different models. The first one, an informed model, characterized by a more traditional workflow, consists of building components based on design drawings of the time, aimed at graphically and quantitatively describe the entire artifact. The second one, a partial model of the only vertical component of the facade, is based on the canons of the element, and it is structured through algorithms that describe only a part of the architecture. The research wanted to deepen the interoperability of the information coming from the parametric models BIM with those generated by the algorithms.
\end{abstract}

\section{INTRODUCTION}

The architectural survey, understood in its broadest sense as an open system of knowledge [Carta del Rilievo Architettonico, 1999] is articulated in the various research, measurement and analysis activities. Through all of these, it is possible to reach the definition of models aimed at a deeper comprehension of the artifact. The implementation of new digital technologies and software platforms have profoundly altered the processes of instrumental acquisition. Simultaneously, the processes of digital modelling of reconstructive type, together with integrated methodologies allow to relate in an effective way the different graphic and informative attributes deriving from the different activities previously described. In a scenario characterized by increasingly detailed and informed models, in line with the new normative dictates, BIM (Building Information Modeling) approaches are increasingly spreading, mainly associated with the design, construction and management of new buildings, and of HBIM type (Historic / Heritage BIM (Murphy, 2009) referable to artefacts of particular architectural or historical value.

This paper intends to show how it is possible to make these processes more efficient and interoperable for the resolution of the problems of digital restitution of the existing as well as in the planning of a new intervention on the historical architecture. One of the main themes of this work is to incorporate the geometric aspects that characterize a certain type of architecture within a broader rule system, which, in the whole, generate the forms. Therefore, the solution would be to draw the reconstruction of a parametric model, through the application to a selected case study: Palazzo del Lavoro of Pier Luigi Nervi. The identification of some weaknesses of the project is important in order to digitally intervene on them, to prefigure a new life of the artifact in a virtual environment. By doing so, it will be possible to have truthful feedback in the real environment.

The survey phase did not intend to reason on the architecture in its complexity, but rather on the observation of the matrices that regulate the similar geometries between them. The translation of these matrices into mathematical models, following the logic of HBIM and Algorithmic Design (AD), has allowed the creation of two different models. The first one, an informed model, characterized by a more traditional workflow, consists of building components based on design drawings of the time, aimed at graphically and quantitatively describe the entire artifact. The second one, a partial model of the only vertical component of the facade, is based on the canons of the element, and it is structured through algorithms that describe only a part of the architecture.

After a critical analysis of the BIM process used for the representation, archiving and sharing of the collected data, the research wanted to deepen the interoperability of the information coming from the parametric models BIM with those generated by the algorithms.

\subsection{Workflow progress}

Today's and avant-garde surveying techniques produce extremely accurate 3D data sets. Thanks to these technological advances, the 3D models are today usable in many fields such as scientific and architectural studies and it is possible to generate images and digital environments capable of illustrating constructed scenarios, developing 3D models that allow immersive virtual explorations. However, despite the digital acquisition and reconstruction procedures are constantly updated, they require sectorial and specific instrumentation. Moreover, unfortunately, processes of elaboration and modeling employ of a conspicuous amount of time. Buildings are almost

\footnotetext{
* Although the contribution was conceived jointly, Massimiliano lo Turco is the author of "Introduction", Andrea Tomalini is the author of "The case study" and "Conclusion"
} 
always characterized by complex geometries which cannot be reproduced optimally by instruments for HBIM. Moreover, modeling architectural details is not easy and fast even with specific modeling tools. It is possible to argue that an approach based on a model capable of adapting to pre-established parameters would optimize the modeling process. As part of this research project, we propose to automate the phase of the modeling process, working mainly on the external vertical infill element: the curtain wall of the Palazzo del Lavoro. What we intend to demonstrate is the potentiality of this approach compared to the more conventional method based on BIM instrumentation. We worked mainly on the geometric relationships of the architectural element, which are highlighted by the structural elements such as ritti ${ }^{1}$ and uprights. The main objective is to highlight how it is possible to parameterize the generation of 3D models of difficult reproduction through BIM tools that can relate different scenarios to each other, through the development of algorithms for 3D virtual reconstruction. It is of fundamental importance to underline how the current research project is a first experiment and represents only an example, which was chosen in order to draw the attention to the state of abandonment of the project case. We work on the curtaiwall with the aim of evaluating the potential of our approach by finding new configurations that are historically and visually congruous but which may suggest a reuse of the building. The relevance of such experiment lies in its ability to carry out two main functions: as modeling tool for a faithful 3D model, and therefore an instrument of restitution; and as study tool for new solutions for a possible refunctionalization, thus a project tool. Instead of modeling the EX NOVO building, we propose a method based on the writing of algorithms. These algorithms should be able to generate parametric objects in which the elements are first created following the logic that regulates them and then adapted either to the measured data or to the new requirements foreseen for the building. This method allows the user to model the architectural element and subsequently make the required adjustments ensuring greater accuracy. These adjustments can be based on data sources such as plans and photographs or be based on new design requirements. After introducing the case study, the principles underlying the proposed method will be listed. In the following parts the modeling phases are described, explaining the differences between traditional and algorithmic modeling. In the last paragraph, instead, the conclusions and future expectations are described.

\section{THE CASE STUDY}

\subsection{Palazzo del lavoro}

In 1961, to commemorate the centenary of the Unification of Italy, Turin inaugurates the International Labor Exposition. The area destined for the event, located in the southern suburbs of the Piedmontese Capital, was designed by Giò Ponti. The architecture of the exhibition pavilions can still be seen inside it: Palazzo del Lavoro, designed and built by Pier Luigi Nervi, stands out for its severity and size.

Palazzo del Lavoro is a virtuous example of dialogue between architectural and structural innovation, widely published in the

\footnotetext{
${ }^{1}$ ritti are special pedestals external to the curtain wall, they are not structurally identifiable as pillars or uprights. They have been designed to react only to the action exerted by the wind on the curtain wall and to the limited axial action due to their own weight. Their external profiling is closely linked to aesthetic needs.
}

major magazines of the time for its ability to optimize material and construction methods using rigorous forms.

The building is characterized by an innovative structure. In fact, the project consisted in the creation of 16 autonomous variablesection mushroom/pillars elements with a side of $40 \mathrm{~m}$ connected to each other by skylights.

They reflect its dimensions, on which Bruno Zevi had already spent some criticisms, as they discouraged his reuse. Palazzo del Lavoro covers a square plan of about 25000 square meters, $650000 \mathrm{mc}$. The roof is supported by 16 impressive 26 meterhigh-ferrocement pillars whose cross-shaped base is $5 \mathrm{~m}$ and at the top of which the metal beams that support the roof are bolted. The elevations, 12160 square meters, are characterized by a curtain wall supported by 12818.6 meter-high ritti among which, with the exception of those located in the North, oriented sunshade slats were welded.

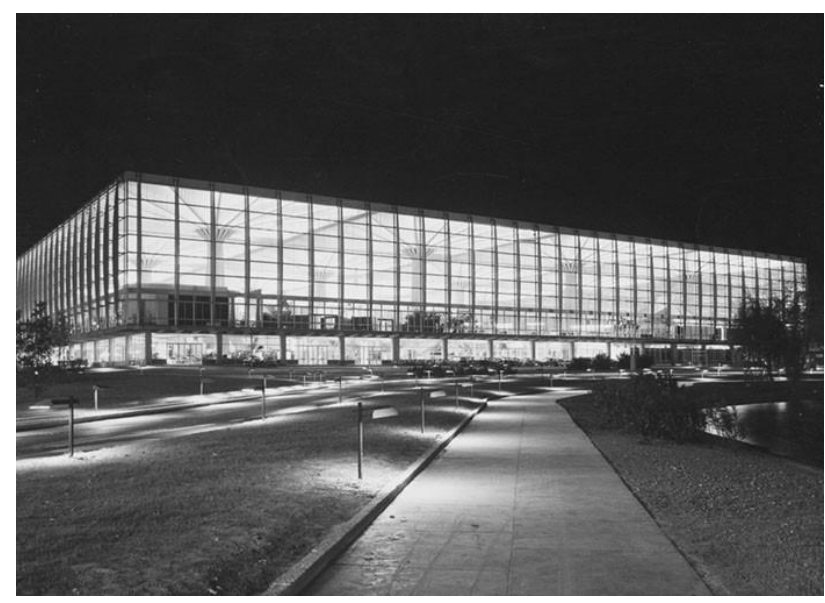

Figure 1: Palazzo del lavoro, night view, 1961. Archivio Storico Fiat

Its construction was also remarkable, lasting only 10 months. The construction site, which began in February 1960, ended in December of the same year. To respect the delivery times, Nervi resorted to the prefabrication of the greatest number of elements, giving as a result an optimization of construction site times. As for the mushroom-shaped pillars of the roof, first the perimeter ones were built, in order to proceed from the first months to the construction of the perimeter tunnel with isostatic ribs. It was subdivided into modules formed in the same formwork that, once disarmed a module, was moved to make the next one. The ritti were raised on them "articolati cardinalmente al piede con amarro sulle opere cementizie e con biellette orizzontali in sommità, fissate alle travi perimetrali metalliche di bordo dei riquadri di copertura. In tal modo i montanti e le vetrate ad essi affidate non risentono praticamente l'effetto termico ed elastico sia delle pareti che della copertura."(Covre, 1961).

Unfortunately, once the Exposition ended, it was not possible to find a permanent function that could occupy these spaces. In fact, excluding some demonstrations and a brief settlement of the BIT, then of the Region, then again of the Faculty of Economics and of the Police Headquarters, it remained unused until today. The structure, as a consequence of this long period of abandonment, is crumbling. In 2007 the ownership of Palazzo del Lavoro became private and since then a proposal has been presented for its reuse in a luxury shopping center. To date it still pours into a state of neglect. 


\subsection{The methodological principles}

Regarding the built heritage, there are various methods for acquiring 3D data of existing buildings, from which point clouds can be used as metric database not yet interpreted. By using these techniques, it is therefore possible to automatically generate new numerical data such as dimensions or new geometric data as lines or 3D primitives.

This new data allows the user to gather precise information which is necessary to model the main parts of a building. However, for objects of large dimensions or particularly articulated and complex in their morphology, a large number of images and/or corresponding points are indispensable, thus increasing the time devoted to the modeling process. As already mentioned above, this type of survey brings with it a lot of energy and cannot in any way be outside the use of specific equipment, as far as modeling is concerned, moreover, it is not always possible to quickly obtain precise and usable BIM models. We believe that it may also be stimulating to consider new approaches based on the study and then on the writing of the criteria that contribute to generate the chosen architecture. We consider that this new approach can better address the geometric complexity of certain architectural elements.

We think that the usefulness of parametric objects goes beyond the virtual reconstruction of the existing built heritage and it may prove to be an interesting tool for the architectural designer. It can be exploited with the aim of exploring new architectural forms within reuse projects or within virtual worlds. arranged following rules described in a second algorithm. To do this, the potentialities expressed by the VPL languages are being studied and evaluated. The purpose of this language in primis is to contribute to the maximum optimization of the timing necessary for the modeling process. In secundis, the purpose is to allow the parametric transposition of any type of shape. In this case, the application will take place through virtual 3D reconstruction projects. The elements that make up this language are not always suitable for the construction of existing architectures. Once the case study of the Palazzo del Lavoro curtain wall was selected, based on the archival drawings, we identified the relationships that govern the proportions. This analysis phase was based exclusively on the observations we conducted and carried out and it did not refer to the quotas or measures in the archive documents. Our case study, Palazzo del Lavoro by Pier Luigi Nervi, is a historically fundamental building for Italian architecture, but it is part of modern architecture. Its construction elements from which it is made are often unique and not connected to the BIM families or elements present or implementable in the databases available to the software. For this reason, if the research was able to create a set of clusters suitable for the modeling of components of historic buildings capable of being inserted into BIM instruments, it would undoubtedly lead to the emergence of widely promising scenarios.

\subsection{The construction of parametric components}

As anticipated, our goal is to tackle the problem of VPL

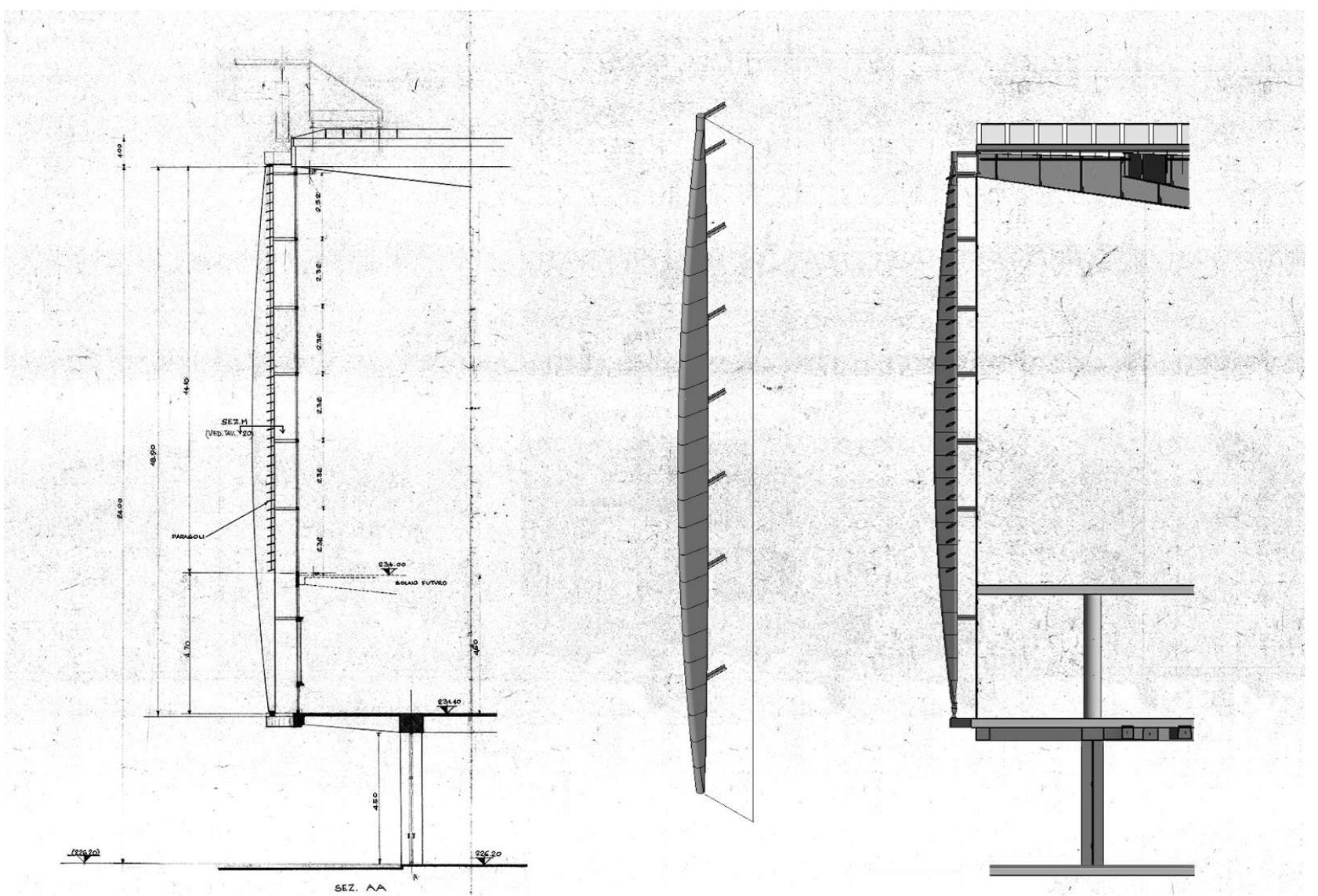

Figure 2: comparison between the Nervi's layout Detail Prospectus n16, Fiat Engineering archive, (on the left) with a vertical section of the BIM model and detail of the Ritto family (in the center and on the right)

We favor a new approach that requires the creation of algorithms that generate $3 \mathrm{D}$ parametric objects that are then modeling integrated with HBIM's tools of architectural details characterized by a high level of geometric complexity with 
respect to the elements used in contemporary architecture. Setting this perspective as a starting point, we have studied the archival drawings concerning Palazzo del Lavoro. In this study we have identified those elements that can be easily modeled within the BIM software and which would have required more attention. As for the mushroom pillars and the ground floor, it was possible to proceed with traditional methods. For the curtain wall, instead, in view of future studies and interpretations, it was necessary but also useful to model it using VPL languages. This dualism is an indispensable aspect to fully understand the fundamental objective of the method proposed by us. It does not want to replace the traditional modeling methods, but to support them and integrate them where there are gaps or difficulties in the execution in order to better correct the result obtained.

\subsection{Traditional modeling:}

The model with BIM tools was executed with the help of Autodesk Revit software. The following are two examples of elements modeled using this software. The first one is the pillar that characterizes the Palazzo del Lavoro, having a lower degree of complexity and it is faster to make. The second one is the ritto, which was also modeled following the proposed new approach.

Parametric component "Structural pillar"

For the realization of the structural pillars it was necessary to start from the family model "structural pillar". In this way, once the pillar will be created and positioned within the project, there will be a correspondence with the analytical model.

The pillar is a geometric ruled surface obtained from the connection of a cross-shaped section at the base and a circular

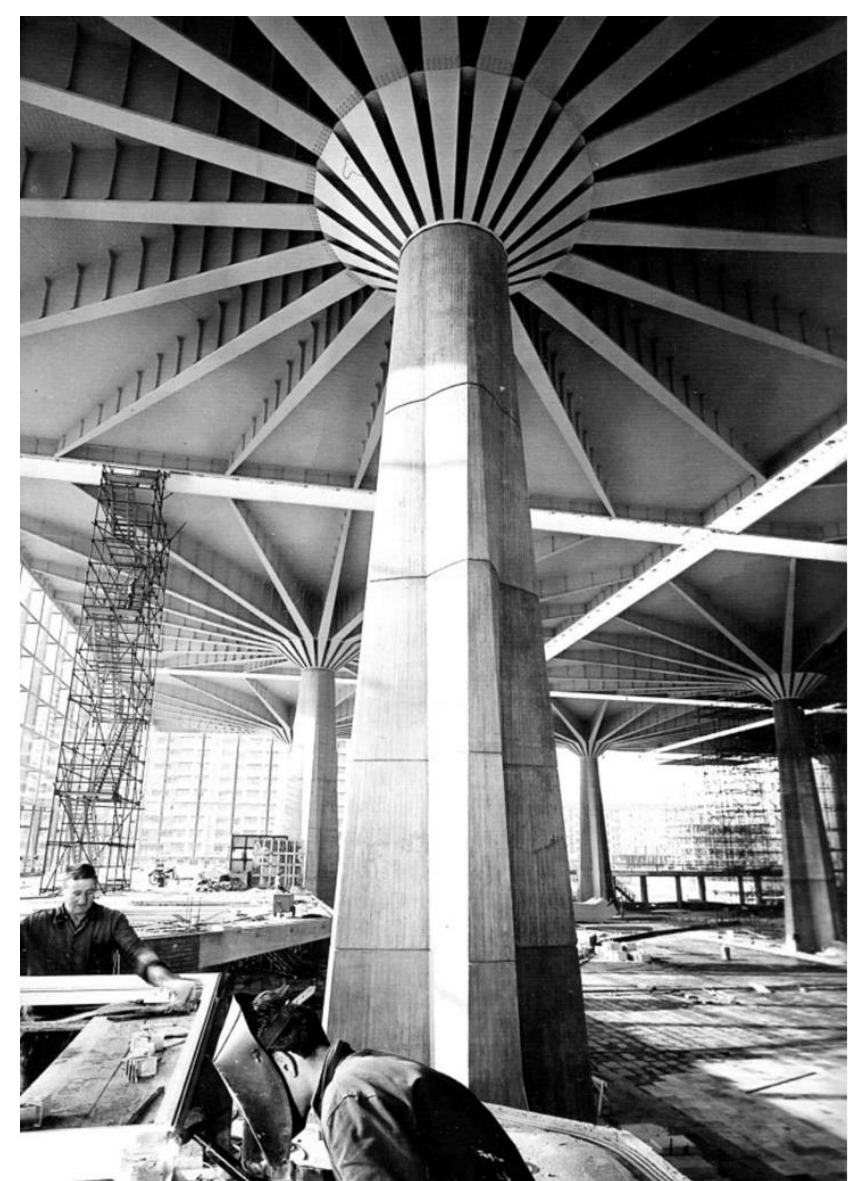

section at the top. In order to be able to model the pillar, it was necessary to draw five equidistant intermediate reference planes, in addition to the reference levels, on which the sections were positioned subsequently.

Although the pillar in question is a unique object of its kind, the choice to reproduce it by parameterizing its sections was dictated by the desire to create a family that can be reused during the preparation of a potential redevelopment project. In this regard, it has been essential to take note and study the drawings of the executive project of the element itself. Starting from the executive layout of Nervi, it is possible to see how the definition of the intermediate sections is the geometric result of the division of the joining lines of the two end sections. Thus, it was possible to define correlations between the measurements of the two sections at the ends, called sections "1" and "7", and the five intermediate sections. These can be changed automatically if the size of one of the sections changes.

Once the dimensional parameters of each section were defined and Nervi's executive drawings were analyzed, it was enough to calculate the surface between them using the sections in the right sequence as "initial shape" and "final form". The result obtained is a pillar with the same geometric characteristics as the original.

The editable parameters in this case are:

section diameter 1 (it is the highest circular one) section diameter 7 (it is the cruciform lower one) section width 7

depth of the arms of the cross (section 7)

The height is calculated automatically based on the anchorage plans marked in the "Base Level" and in the "Upper Level". Family "perimetric Ritto"

The perimeter ritto is the element that takes care of all the actions acting on the facades. It then discharges the weight on

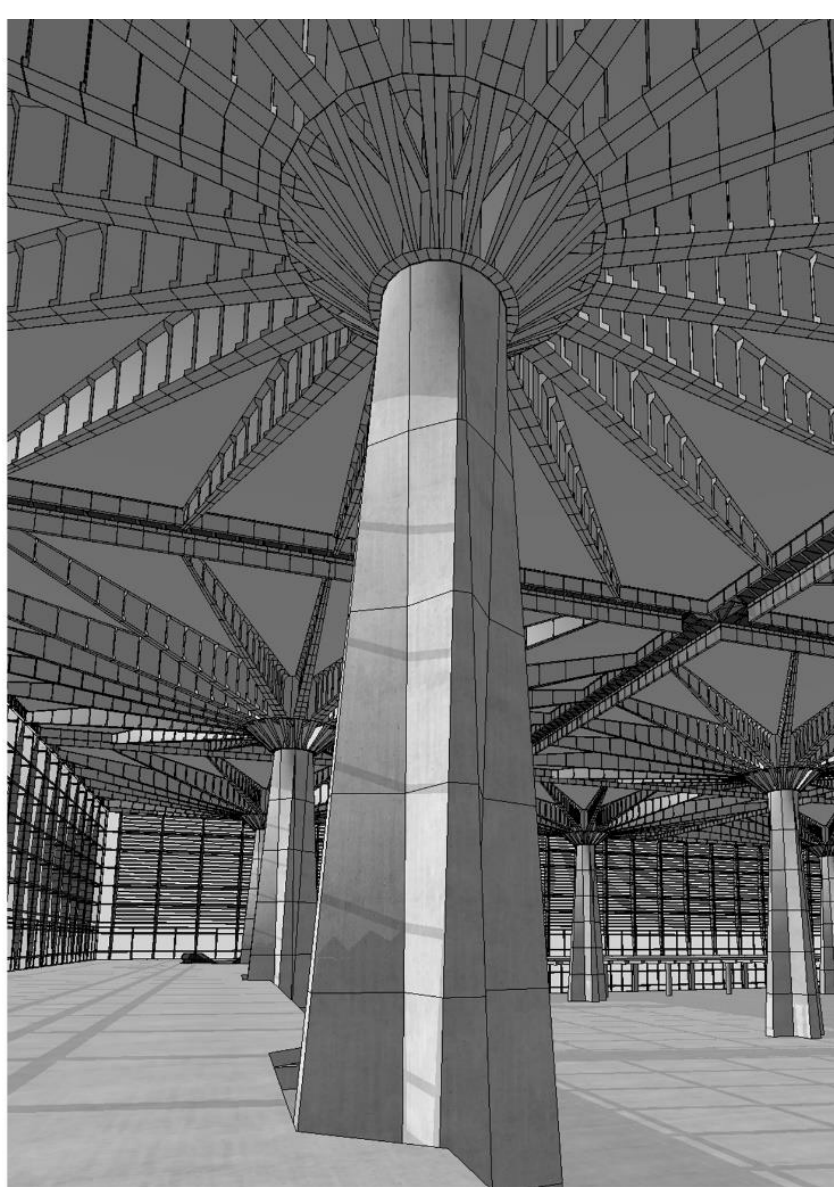

the floor of the perimeter balcony on the First Floor through a

Figure 3: comparison between a a photo of the construction site, Sergio Poretti's archive Rome (on the left), with a perspective view of the BIM model (on the right) 
cardan joint; for this reason, it was necessary to model the joint separately from the ritto itself.

The choice of the family model from which to start for these two elements was not trivial. Additionally, it constituted an important starting point for study and analysis. In fact, they do not fit neatly into any of the macro-categories that Revit considers while creating constructive elements. In light of these considerations for the joint, it was decided to start from a generic model and then go on to modify the category of the family in "structural connection". As for the ritto itself, it was decided to use the "structural pillar" family model, although it is not a real pillar.

During the development of the cardan joint geometry, it was decided to simplify the geometry of the element. In fact, it will not be the object of particular investigation in the following phases.

For the modeling of the ritto it was necessary, as for the pillar, to divide the whole element into a defined number of sectors. These have been studied on the metal carpentry tables in order to realize a more likely geometry than the existing one. As before, it was enough to calculate the surface using the sections in the right sequence as "initial form" and "final form". In this case, given the uniqueness of the element and the large number of sectors necessary to represent the upright, it was decided not to parameterize the dimensions of the sections. Other dimensions can be edited as: the total height of the element, the distance between the anchoring elements to the curtain wall and the length of the connecting rod to the roof.

The parameters in this case are: total height of ritto

height at which the upper link is located

width of the connection element

thickness of the connection element

length of the connection element

In this case there is no need for the lower and upper double anchoring but only the lower one since the height is not deducted automatically but is an internal parameter.

In both cases, the objective of creating two parametric families that can be used later also in the design phase, since they are parameterized, was achieved. For what concerns the second case, the considerable complexity of the element is divided into an excessive number of segments to be able to derive reasonable parameters for the update. Thus, it has not been possible the complete parameterization. In fact, when attempting to edit the element with respect to its height, it will be lengthened by not respecting the proportions or the curvature of the ritto (Paris,
2016).

\subsection{VPL modeling:}

The model with VPL tools was made with the help of the Grasshopper3D software, application of the Rhinoceros3D software. Below are two examples of elements modeled using this software: first, there is the glazed element of the curtain wall, coupled with, where present, the darkening element. These have a lower degree of complexity and are faster to make than the second one. Then, there is the ritto, which, was also modeled following the previously explained BIM approach. Family "glass element of the curtain wall"

The curtain wall would also have been well suited for modeling using BIM-type tools in fact, there are families related to its morphology. It was essential to take into consideration the fact that the horizontal and vertical uprights of the curtain wall are specially connected to the perimeter ritti and to the darkening elements. For these reasons it was more appropriate to first proceed with the writing of the subdivision rules of the element itself. The outputs of this algorithm will be indispensable for positioning the uprights and the darkening elements.

The algorithm needs as initial input a rectangular surface drawn counter-clockwise (with the normal outgoing) and as first vertex the lower one to the left is inserted. The surface is divided into two parts: the lower one, the one on which the slats of the darkening system will not be inserted, it represents the first floor; the second one, instead, which is the part of the remaining elevation. These surfaces are immediately broken down into vertices and sides. The sides are measured and, on the basis of these measurements, the pitch of the horizontal and vertical uprights is drawn and the vertices are used to understand the orientation of the sides. On the lines that identify the pitch of the uprights, some profiles have been extruded, which can also be modified.

Although the final result is visually very close to what we could have obtained using a BIM application, the information generated with the used methodology is different. The potential of this model is its ability to be modifiable through the movement of a slider. The algorithm, moreover, as it has been written, does not allow a radical distortion of the initial conformation of the case study. It guarantees the possibility of intervening through various approaches, always with total respect for the proportions desired by the original drawing by Pier Luigi Nervi.

The editable parameters in this case are:

\section{- height of the first floor}

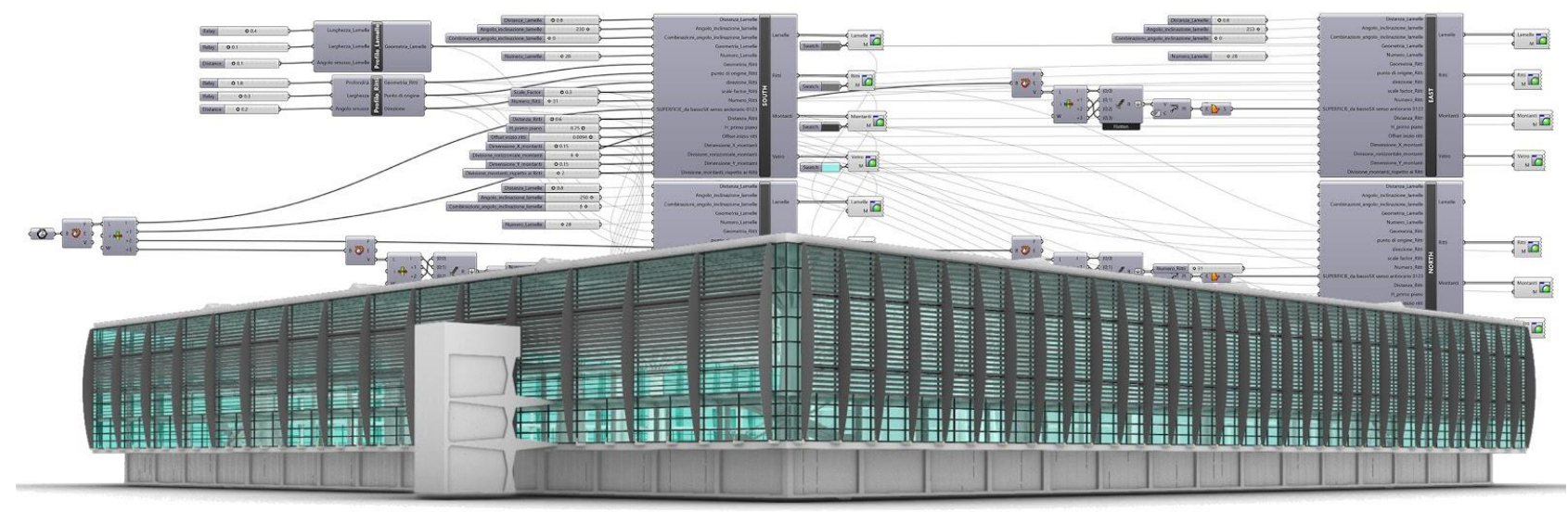

Figure 4: the merging of the algorithmic model of the curtainwall and the BIM model of the remaning part of the building. The south facade (on the right) re-proposes the current state, the west facade (on the left) demonstrates the potential of the code in customi 
horizontal division of uprights

vertical upright division

uprights depth

uprights width

Family "Perimeter Ritto" and "darkening element"

The perimeter ritti family needs an initial geometry that describes the section at the base of the ritto. This is described in a separate algorithm so that it can be edited more easily. The profile can be modified along its extrusion by scaling or deforming the initial section. The height of the ritti is calculated automatically, based on the initial surface. The pitch of the uprights is editable through a slider, more or less, one each vertical upright. The connections between ritti and uprights are automatically made at the intersection of the vertical upright to which the ritti refers and the horizontal uprights that intersect it. This same element was also the object of modeling using the Autodesk Revit software, the result obtained is an object that is much more flexible and easier to position within the model.

The editable parameters in this case are:

pitch of the ritti compared to the uprights

offset from the support plane

distance from the reference upright

geometry of the upright

deformation factor of the ritto

height where deformation occurs

Using the height of the ritti and their pitch as output, the algorithm for the slats of the obscuring element was written. The construction is similar to that of the ritti, once the section is inserted it is extruded along a line. In addition, it is possible to modify their inclination. The element was modeled through scripts, not because it is more flexible than a model created with BIM tools, in fact the parameters that can be modified are the same. But because thanks to the proposed method the element is able to adapt automatically to the modifications that are made to the perimeter uprights.

The editable parameters in this case are:

distance between the slats

slat inclination

number of slats

slat geometry

With this type of programming, the VPL, the objects are only displayed by the computer until they are converted within Rhinoceros3D. Until the moment in which the model has not yet been converted from a displayed object to a modeled object, the user can enjoy the possibility of modifying the data by using a slider. After each change, the 3D model is regenerated in real time. In this way the user can check if the modifications he makes produce the expected or desired effects on the model. The objects are organized hierarchically, which means that each element is composed of a set of sub-elements. Just think how the horizontal connections between the ritti and the curtain wall are generated automatically within the family of ritti but cannot be directly edited. It can be deduced how the dimensions of the components are linked together. When the dimensions of the panels that make up the curtain wall vary, these changes are transmitted to the sub-elements; if the height of the initial surface varies, the one of the elements as uprights or ritti is automatically updated. As previously mentioned, to elaborate a $3 \mathrm{D}$ model that represents an architectural element, the user must configure, through the sliders, the parameters that describe it. So, in addition to the advantage of having flexible and ready-tochange models, it is possible to transfer files, which once set correctly, contain the same geometric information but occupy less than half of the space. Consider that the family of the upright in proprietary .rfa file has a weight of $540 \mathrm{~KB}$ while, the same data, on .gh files, have a weight of only $157 \mathrm{~KB}$ (Chevrier et al., 2010).
Referring to a wider workflow, compared to the reconstruction in digital environment of existing buildings, it can be said that the VPL programming, thanks to its OpenSources soul, offers an unlimited library of plug-ins that do not stop at the sole control of the geometries but unblock other numerous functions. Through these tools, the gap in the development of a project between the initial phase and the construction of a BIM model is bridged. As anticipated, the plug-ins are multiple, they do not only refer to the purely architectural field, and they unblock control functions previously not accessible to the "nonprogrammers" who can now guide the architect during the decision-making process.

\section{CONCLUSIONS}

This test, albeit limited to the only vertical darkening component of Palazzo del Lavoro, demonstrates how a new parametric approach to the construction of digital models and the management of informed HBIM models is possible.

A different analysis of the geometry that compose the architectural element makes possible to identify the grammar that regulates the shapes of each element and, consequently, facilitates the translation of these geometries in a digital environment and the subsequent communication between different models.

During this activity it immediately emerged how the AD-based models are able to handle different variables in a short time. In fact, the combination of the processes of HBIM and algorithmic Design, within a process of recovery or reuse of an artifact, allows to address the criticalities of complex case studies in a more dynamic way with a consequent saving of time in the elaboration of possible solutions appreciating and designing the variable movement. It is evident that the use of objects written with algorithms leads to a considerable saving of time by optimizing the modeling process.

This interesting hybridization between $\mathrm{AD}$ and HBIM, if structured effectively, could suggest in the future the use of alternative workflows for obtaining informed and flexible historical models, usable in the preliminary project phase, also useful to prefigure subsequent reuse or recovery interventions.

It should be emphasized that, thanks to the continuous evolution of these systems, the interoperability processes between $\mathrm{AD}$ and HBIM are approaching the two workflows by offering new perspectives in both fields. This new and unique workflow offers tools that overcome the simple bi-directional transfer of geometries. Thanks to the new interoperable processes it is possible to translate geometric shapes into BIM elements while preserving the opportunities derived from the $\mathrm{AD}$ world.

Although our experiment has led to promising results, the limits of this approach are still many. In future developments it would be interesting to improve its flexibility by creating clusters that can adapt not only to the chosen case study. Furthermore, it would be interesting to study a method that guides the user in the initial collection of data relevant for the written algorithms

\section{ACKNOWLEDGEMENTS}

The authors would like to thank the Architect C.Chiorino for his willingness to share numerous drawings regarding Palazzo del Lavoro. 
Tomalini would like to thank Prof. M. Lo Turco and Phd. M. Calvano for their precious collaboration that it was essential to conduct investigations into the case study.

Tomalini would also like to thank his colleagues A.Trovato and E.Tomalini for their technical support.

\section{REFERENCES}

Chevrier, C., Charbonneau, N., Grussenmeyer, P., and Perrin, J. P., 2010. Parametric documenting of built heritage: 3D virtual reconstruction of architectural details. International Journal of Architectural Computing, 8(2), pp. 135-150.

Covre G., 1961. Nuove costruzioni, Il Palazzo del Lavoro alla esposizione <<Italia 61>> di Torino, Le pareti vetrate. Costruzioni metalliche, 3 , pp.141

Murphy M., Mcgovern E., Pavia S., 2009. Historic building information modelling (HBIM). Structural Survey, 27(4), 311327.

Paris L., Wahbeh W., 2016. Rilievo e rappresentazione delle geometrie parametriche per l'HBIM. Disegnarecon, Vol. IX, n.16, pp. 12.1-12.9. 